\title{
American Academy of Dental Sleep Medicine Position on the Scope of Practice for Dentists Ordering or Administering Home Sleep Apnea Tests
}

David Schwartz, DDS ${ }^{1}$; Michael Adame, DDS²; Nancy Addy, DDS³; Michelle Cantwell, DMD4; James Hogg, DDS5; Nelly Huynh, PhD6; Paul Jacobs, DDS 7 ; Mitchell Levine, DMDं Kevin Postol, DDS ${ }^{9}$; Rosemarie Rohatgi, DMD ${ }^{10}$

${ }^{1}$ North Shore Family Dentistry, Skokie, IL; ${ }^{2}$ Adame Dental Sleep Medicine; ${ }^{3}$ Snoring and Sleep Apnea Dental Treatment Center, Leawood, KS; ${ }^{4}$ Wellspan Pulmonary and Sleep Medicine, Lancaster, PA; ${ }^{5}$ Carolina Smiles Family Dentistry, Brevard, NC; ${ }^{6}$ Faculty of Dentistry, Universite de Montreal, Montreal, Canada; ${ }^{7}$ upper Peninsula Sleep Dentistry, Escabana, Ml; ${ }^{8}$ Department of Orthodontics, University of Tennessee Health Science Center, Memphis, Tennessee; ${ }^{9}$ Sleep Disordered Dentistry, Ballwin, Missouri; ${ }^{10}$ San Diego Sleep Therapy, San Diego, CA

It is the position of the American Academy of Dental Sleep Medicine (AADSM) that it is within the scope of practice for a qualified dentist, defined by the American Dental Association (ADA) as a dentist treating sleeprelated breathing disorders who continually updates his or her knowledge and training of dental sleep medicine with related continuing education, to order or administer home sleep apnea tests (HSATs). Data from HSATs should be interpreted by a licensed medical provider for initial diagnosis and verification of treatment efficacy.

Historically, state dental practice acts have not addressed the dentist's role in using HSATs. It is commonly understood that practice acts are intentionally broad in nature. They tend to be more specific only when prohibiting a practice or use of equipment. Based on this, it is the AADSM's interpretation that it is within the scope of practice for dentists to order and administer HSATs in states where it is not specifically prohibited. For the few states where the use of HSATs is prohibited, dentists should abide by state guidance. The AADSM maintains a list of these states on its website and will be actively encouraging them to reconsider their policies. ${ }^{1}$

There are other medical conditions for which dentists order and dispense medical tests. Dentists screen and perform biopsies for oral cancer. Dentists routinely administer oxygen and anesthesia and prescribe drugs, including controlled substances. In some states, dentists with training provide flu vaccinations. Dentists also routinely take blood pressure and some test hemoglobin A1C levels. Given the public burden of obstructive sleep apnea (OSA), dentists must embrace that it is within their scope of practice to order and administer HSATs.

In 2016, the American Academy of Sleep Medicine commissioned a report from Frost \& Sullivan. ${ }^{2}$ This report indicates that there were 29.4 million adults with obstructive sleep apnea, and in $80 \%$ of that group the condition was undiagnosed - costing the United States approximately $\$ 149.6$ billion per year. The same report indicated that OSA is also linked to comorbidities, mental health, productivity, and accidents. It goes on to further explain that the most significant barrier to treatment of OSA is patients' disregard of symptoms and their failure to report them to primary care physicians and that once an individual is screened or inf ormed about OSA, a significant financial and personal time investment is often necessary to address the problem. New studies published in 2019 indicate that approximately 54 million adults in the United States have sleep apnea. ${ }^{3}$ If $80 \%$ of these adults also have undiagnosed OSA, there could be as many as 43 million adults with undiagnosed OSA.

In 2017, the ADA recognized that dentists should play an essential role in addressing the public burden of OSA. ${ }^{4}$ In their policy, the ADA suggests that all dentists screen patients for OSA as part of a comprehensive medical and dental history and refer as needed to the appropriate physicians for diagnosis. The policy indicates that dentists may use HSATs to define the optimal target position of the mandible.

By building on the ADA policy and recognizing that qualif ied dentists have the training and education necessary to order or administer HSATs, qualified dentists can provide a more streamlined and cost-effective model of care. A short algorithm outlining this model of care is shown in Figure 1. Communication and collaboration with physicians are key in this process. In this model of care, qualified dentists screen patients for sleep apnea. If patients are at risk and appropriate candidates for HSAT, the qualified dentist orders or administers the HSAT directly from his or her practice. Patients complete the HSAT. Pertinent patient information and HSAT data are provided to a physician for diagnosis, and, if appropriate, the physician prescribes an oral appliance. The qualified dentist then determines whether the patient is a suitable candidate, and then fabricates and delivers the appliance. After the appliance is at the appropriate therapeutic position, the qualified dentist once again orders or 
administers the HSAT. Pertinent patient information and HSAT data are shared with the physician who verifies treatment efficacy.

This model of care achieves several outcomes:

1. Dentists identify patients at risk for sleep apnea.

2. The process of obtaining a diagnosis for sleep apnea requires fewer appointments, reducing expenses and patient inconvenience while increasing the likelihood of treatment if sleep apnea is diagnosed in a patient.

3. The workload of primary care physicians and board-certified sleep medicine physicians related to ordering and dispensing HSATs is reduced, allowing them to better allocate their resources to the diagnosis and treatment of sleep disorders.

4. The diagnosis of medical diseases and verification of treatment efficacy remains the responsibility of the medical provider.

With the public burden of OSA and technologic advances, new models of care are being implemented at a rapid pace. Patients can now purchase HSATs directly from online sources. It is hard to find an argument against allowing a qualified dentist who will collaborate directly with patients' physicians when patients can order the test directly from the Internet, entirely bypassing their health care providers.

As health care providers who live by the ethical code of "do no harm" and understand the harmful consequences of OSA, we owe it to the public to implement models of care that reduce barriers to diagnosis and treatment, ensure that sleep apnea is diagnosed and treatment efficacy is verified by physicians, and maximize the training and skills of qualified dentists.

\section{CITATION}

Schwartz D, Levine M, Adame M, Addy N, Cantwell M, Hogg J, Huynh N, JacobsP, Postol K, Rohatgi R. American Academy of Dental Sleep Medicine Position on the Scope of Practice for Dentists Ordering or Administering Home Sleep Apnea Tests. J Dent Sleep Med. 2020;7(4).

\section{REFERENCES}

1. Home sleep apnea tests. American Academy of Dental Sleep Medicine. https://www.aadsm.org/home_sleep_apnea_tests.php. Accessed August 18, 2020.

2. Frost \& Sullivan. Darien, IL: American Academy of Sleep Medicine; 2016. Hidden health crisis costing America billions. Underdiagnosing and undertreating obstructive sleep apnea draining healthcare system. https://aasm.org/advocacy/initiatives/economic-impact-obstructivesleep-apnea. Accessed August 18,2020.

3. Benjafield AV, Ayas NT, Eastwood PR, et al. Estimation of the global prevalence and burden of obstructive sleep apnoea: a literature-based analysis. Lancet Respir Med. 2019;7(8):687-698. doi:10.1016/S22132600(19)30198-5

4. Policy on Dentistry's Role in Treating Obstructive Sleep Apnea, Similar Disorders. American Dental As sociation. ada.org/sleepapnea. Accessed August 18, 2020.

\section{SUBMISSION AND CORRESPONDENCE INFORMATION}

\section{Submitted in final revised form August 28, 2020.}

Address correspondence to: David Schwartz, DDS; Email: dschwartz@aadsm.org

\section{DISCLOSURE STATEMENT}

All authors are members of the AADSM Board of Directors. Dr. Schwartz declares investments in Prosomnus Sleep. 
Figure 1. Model of Care

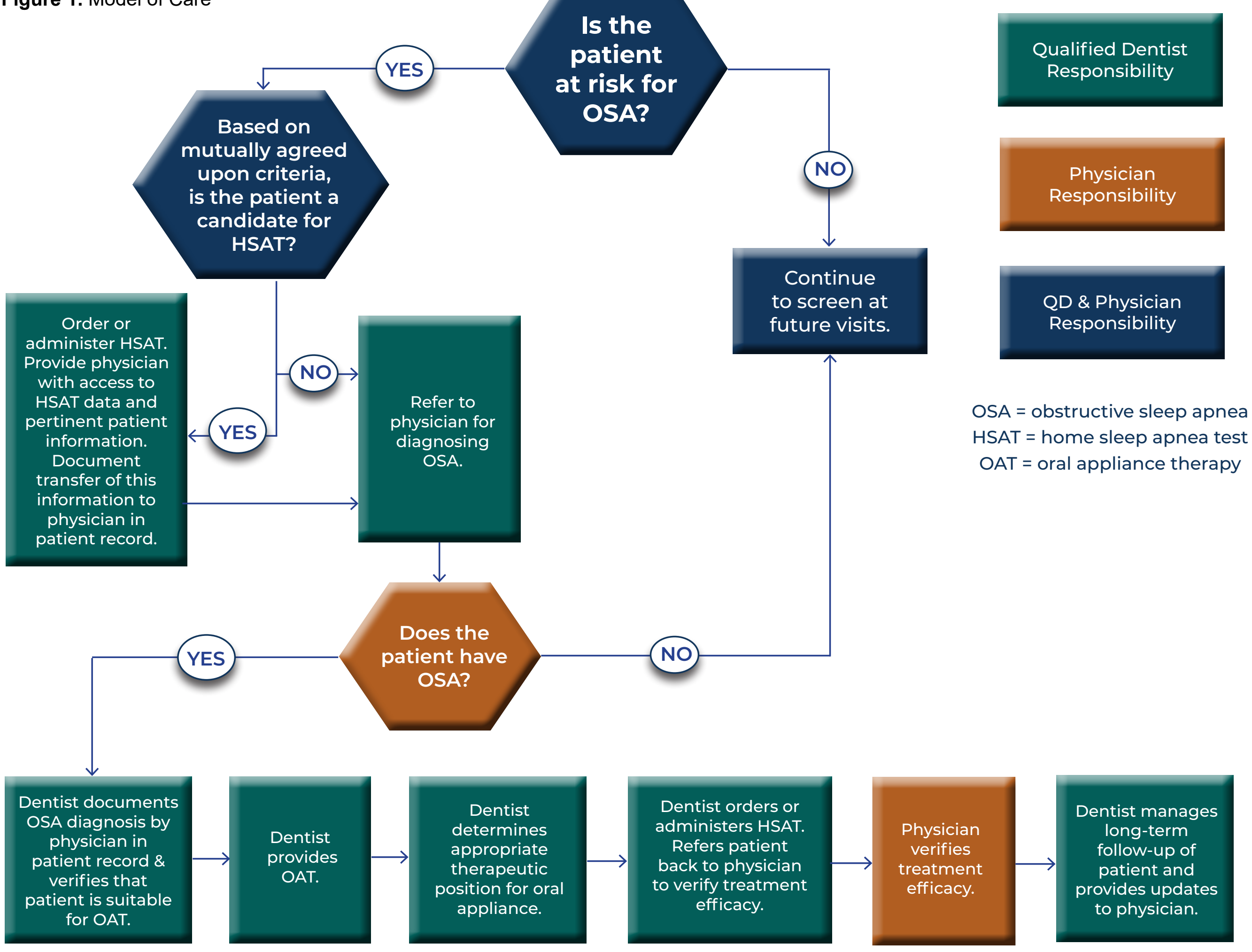

